\title{
INDUCIBLE CLINDAMYCIN RESISTANCE IN STAPHYLOCOCCUS AUREUS ISOLATES AND ITS CORRELATION WITH MULTIDRUG RESISTANCE: A STUDY FROM NORTH INDIA
}

Shivani Tyagi ${ }^{1}$, Aroma Oberoi ${ }^{2}$

${ }_{1}^{1}$ Senior Resident, Department of Microbiology, G. B. Pant Hospital, New Delhi.

${ }^{2} H O D$, Department of Microbiology, Christian Medical College \& Hospital, Ludhiana, Punjab.

\section{ABSTRACT}

\section{CONTEXT}

In Staphylococcus aureus strains with inducible resistance antibiotic therapy with clindamycin may select constitutive erm gene mutants in vivo leading to clinical failure. Therefore, it is imperative to do a simple 'D-test' to detect isolates with inducible clindamycin resistance. Very few studies are available to state whether or not this inducible resistance has any correlation with multidrug resistance.

\begin{abstract}
AIMS
To find out the prevalence of inducible clindamycin resistance amongst S. aureus isolates from various clinical specimens and determine its correlation with multidrug resistance.
\end{abstract}

\section{SETTINGS AND DESIGN}

A prospective study done in the Department of Microbiology in a tertiary care hospital for a period of ten months.

\section{METHODS AND MATERIAL}

All strains of S. aureus isolated from various clinical specimens were subjected to Antibiotic Susceptibility Testing as per CLSI guidelines and methicillin resistance was detected using cefoxitin. All the erythromycin resistant and clindamycin susceptible isolates were further subjected to 'D-Test.'

\section{STATISTICAL ANALYSIS USED}

Data was entered in Microsoft excel and analysed using SPSS version 16. Frequencies and proportions were done. Chi square test was the test of significance.

\section{RESULTS}

Out of 1153 S. aureus strains isolated $49.8 \%$ were methicillin resistant and 50.2\% were methicillin susceptible. Prevalence of inducible phenotype, MS phenotype and constitutive phenotype was $18.4 \%, 22.1 \%, 18.4 \%$ respectively. Resistance to penicillin, amoxicillin-clavulanic acid, ciprofloxacin, sulphamethoxazole-trimethoprim was $92.1 \%, 47.8 \%, 43.4 \%$ and $49.3 \%$ respectively, whereas none of the isolates showed resistance to vancomycin, teicoplanin and linezolid. About $22.8 \%, 41.6 \%$ and $82.9 \%$ isolates with inducible phenotype, MS phenotype and constitutive phenotype respectively were multidrug resistant.

\section{CONCLUSION}

Prevalence of multidrug resistance is least likely with inducible clindamycin resistance than with other phenotypes.

\section{KEYWORDS}

Staphylococcus Aureus, Inducible Phenotype, Multidrug Resistance.

HOW TO CITE THIS ARTICLE: Tyagi S, Oberoi A. Inducible clindamycin resistance in staphylococcus aureus isolates and its correlation with multidrug resistance: a study from north India. J. Evolution Med. Dent. Sci. 2016;5(16):749-752, DOI: $10.14260 /$ jemds/2016/173

\section{INTRODUCTION}

Methicillin resistance in Staphylococcus aureus (S. aureus) a recognized nosocomial and community acquired pathogen worldwide is an escalating problem today. ${ }^{1}$ The MacrolideLincosamide-Streptogramin B (MLS $\mathrm{B}_{\mathrm{B}}$ ) family of antibiotics serve as an alternative therapeutic agent to treat such $\mathrm{S}$. aureus infections with clindamycin being the preferred agent. ${ }^{2}$

Financial or Other, Competing Interest: None.

Submission 13-01-2016, Peer Review 07-02-2016,

Acceptance 12-02-2016, Published 23-02-2016.

Corresponding Author:

Dr. Aroma Oberoi,

C/o Department of Microbiology,

Christian Medical College \& Hospital,

Ludhiana-141008, Punjab.

E-mail: draromaoberoi@yahoo.com

DOI: $10.14260 /$ jemds/2016/173
However, the widespread use of MLS $в$ antibiotics has led to an increase in the number of staphylococcal strains acquiring resistance to these antibiotics as well.3,4 A common mechanism by which staphylococcal strains acquire resistance to $\mathrm{MLS}_{\mathrm{B}}$ antibiotics is the target site modification mediated by erm gene products. ${ }^{5}$ The erm gene can be expressed either constitutively (Constitutive $\mathrm{MLS}_{\mathrm{B}}$ phenotype) or inducibly (Inducible MLS $S_{B}$ phenotype), where the gene product is either produced always or only in the presence of an inducing agent like erythromycin respectively. Another mechanism of resistance that affects only macrolides and type $B$ streptogramins is by efflux by msr A gene (MS phenotype). $6,7,8$ In vitro, S. aureus isolates with constitutive resistance show resistance to erythromycin and clindamycin and isolates with inducible resistance are resistant to erythromycin, but appear susceptible to clindamycin. 
In vivo antibiotic therapy with clindamycin may select constitutive erm gene mutants. ${ }^{9}$ which may lead to clinical failure.10,11,12 Staphylococcal isolates with msr A-mediated efflux mechanism also appear erythromycin resistant and clindamycin susceptible by in vitro tests, but such isolates do not become clindamycin resistant in vivo. A simple in vitro test known as disk diffusion induction test (D-Test) can distinguish between the staphylococcal strains with inducible ermmediated resistance from those with msr A mediated resistance (Figures: 1 \& 2).2,10,13 In vitro routine susceptibility tests may fail to detect inducible clindamycin resistance, thus necessitating the need to detect such resistance by the ' $\mathrm{D}-\mathrm{Test}$ on a routine basis, so as to ensure safe and effective use of clindamycin. Because very few studies have been done correlating inducible clindamycin resistance and multidrug resistance, this study was done to find out the prevalence of inducible clindamycin resistance amongst $\mathrm{S}$. aureus isolates from various clinical specimens and determine its correlation with multidrug resistance.

\section{MATERIALS AND METHODS}

This prospective study was conducted in the Department of Microbiology in a tertiary care hospital for the duration of 10 months and included all isolates of $\mathrm{S}$. aureus isolated from various clinical specimens like pus, urine, wound swab, aspirates, blood, respiratory secretions and sterile fluids, etc. (Figures: 3 \& 4). The isolates were first identified as S. aureus by standard biochemical techniques. ${ }^{14}$ and then subjected to Antibiotic Susceptibility Testing by Kirby Bauer's disk diffusion method on Mueller Hinton Agar plates using erythromycin $(15 \mu \mathrm{g})$, penicillin $(10 \mathrm{U})$, clindamycin $(2 \mu \mathrm{g})$, vancomycin $(30 \mu \mathrm{g})$, teicoplanin $(30 \mu \mathrm{g})$, amoxicillin-clavulanic acid $(20 / 10 \mu \mathrm{g})$, ciprofloxacin $(5 \mu \mathrm{g})$, linezolid $(30 \mu \mathrm{g})$ and sulphamethoxazole-trimethoprim $(1.25 / 23.75 \mu \mathrm{g})$ as per CLSI guidelines. ${ }^{15}$ Methicillin resistance was detected using cefoxitin $(30 \mu \mathrm{g})$ on Mueller Hinton Agar supplemented with $2 \% \mathrm{NaCl}$ followed by incubation at $35^{\circ} \mathrm{C}$. An inhibition zone of $21 \mathrm{~mm}$ or less around Cefoxitin disk indicated methicillin resistance. ${ }^{15}$ All the erythromycin resistant and clindamycin susceptible isolates were further subjected to 'D-Test' as per the CLSI guidelines by placing an erythromycin $(15 \mu \mathrm{g})$ disk at a distance of $15 \mathrm{~mm}$ (Edge to edge) from clindamycin $(2 \mu \mathrm{g})$ disk on a Mueller Hinton Agar plate previously inoculated with 0.5 McFarland bacterial suspension. Following overnight incubation at $37^{\circ} \mathrm{C}$, if there is (D shaped) flattening of zone of inhibition around clindamycin disk in the area between the two disks, it indicates inducible clindamycin resistance (Figure: 1). ${ }^{15}$

\section{RESULTS}

A total of $1153 \mathrm{~S}$. aureus strains were isolated and included in the study with maximum isolation from pus, wound swabs, tissue and other pyogenic samples 63\% (726) followed by blood 29.3\% (338) and urine samples 4.7\% (54) etc. (Figure: 4). Of these 1153 strains, $49.8 \%$ (574) were methicillin resistant and $50.2 \%$ (579) were methicillin susceptible. On antibiotic susceptibility testing, 59\% (680) isolates were erythromycin resistant which were then subjected to D-Test, where 212 (18.4\%) isolates showed Inducible Phenotype, 255 (22.1\%) isolates showed MS Phenotype and 212 (18.4\%) isolates showed Constitutive Phenotype. Resistance pattern to different antibiotics tested was as follows:
Percentage of isolates resistant to penicillin, amoxicillinclavulanic acid, ciprofloxacin, sulphamethoxazoletrimethoprim were $92.1 \%, 47.8 \%, 43.4 \%$ and $49.3 \%$ respectively. None of the isolates showed resistance to vancomycin, teicoplanin and linezolid. About 22.8\%, 41.6\% and $82.9 \%$ isolates with Inducible phenotype, MS phenotype and Constitutive phenotype respectively were multidrug resistant based on the resistance of an isolate to three or more than three class of drugs.

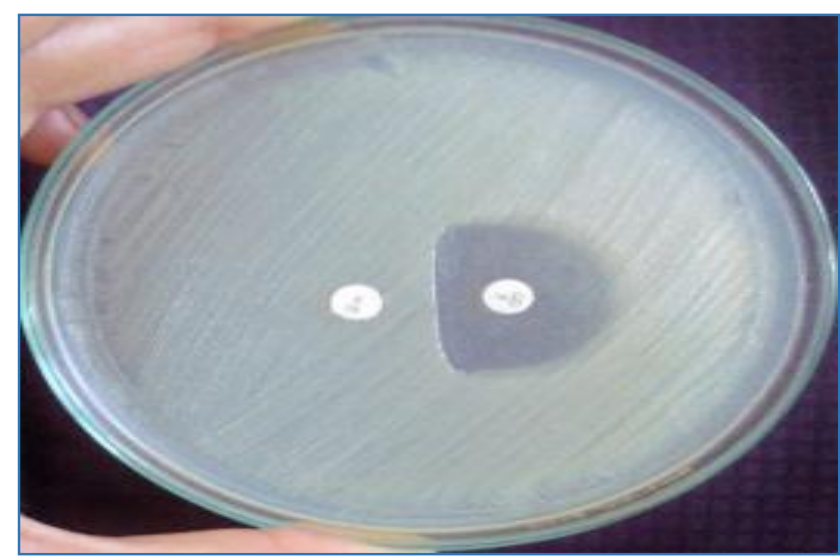

Fig. 1: Inducible MLSB Phenotype: Flattening of the Clindamycin Zone of inhibition Adjacent to Erythromycin Disk (D-Test positive)

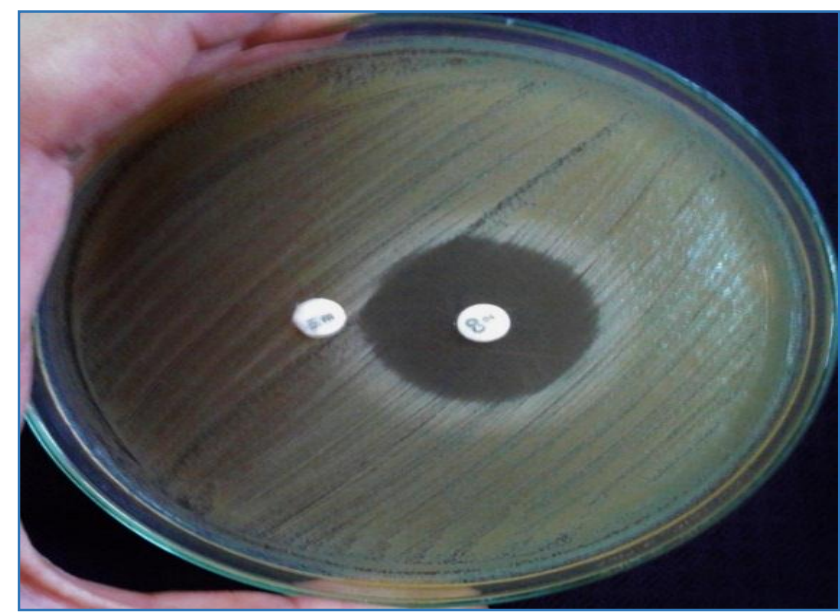

Fig. 2: MS Phenotype: No Blunting of Zone of Inhibition around Clindamycin adjacent to Erythromycin Disk (D-Test negative)

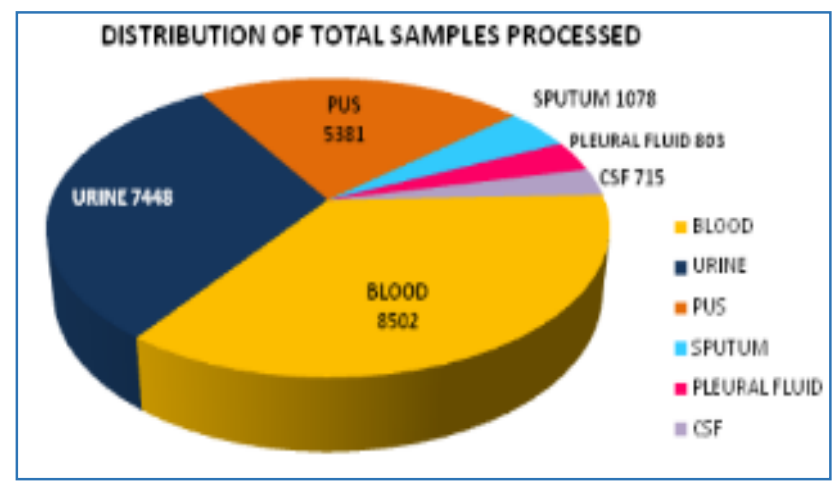

Fig. 3: Distribution of total samples processed 


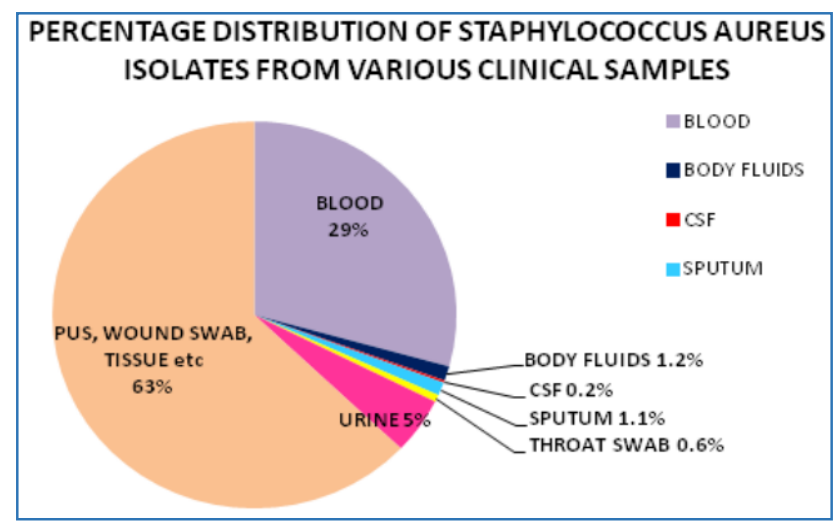

Fig. 4: Percentage distribution of Staphylococcus aureus Isolates from various Clinical Samples

\section{DISCUSSION}

S. aureus is an important nosocomial and community acquired pathogen worldwide. ${ }^{1}$ which can cause both superficial and deep pyogenic infections as well as a number of toxin mediated illnesses. ${ }^{16}$ With the emergence of methicillin resistance in $\mathrm{S}$. aureus, $\mathrm{MLS}_{\mathrm{B}}$ family of antibiotics serve as an alternative therapeutic agent with Clindamycin being the preferred agent. ${ }^{2}$ erm gene expression leading to ribosomal target site modification leads to resistance to these $M_{L} S_{B}$ group of drugs including Clindamycin. This erm gene can be expressed either constitutively or inducibly. In vitro, S. aureus isolates with constitutive resistance are resistant to Erythromycin and Clindamycin and isolates with inducible resistance are resistant to Erythromycin, but appear susceptible to Clindamycin; however, in vivo antibiotic therapy with Clindamycin may select constitutive erm gene mutants. ${ }^{9}$ which may lead to clinical failure.10,11,12 thus necessitating the need to detect such resistance by a simple 'D-Test' on a routine basis, so as to ensure safe and effective use of Clindamycin, only in those patients with truly susceptible strains.

In the present study we included $1153 \mathrm{~S}$. aureus isolates, out of which $49.8 \%$ isolates were Methicillin resistant and $50.2 \%$ were Methicillin susceptible. The findings were in concordance with the observations made by other authors like Deotale et al. (2010). ${ }^{3}$ Gadepalli et al. (2006). ${ }^{17}$ Yilmaz et al. (2007). ${ }^{1}$ and Azap et al. (2005). ${ }^{18}$ Unlike our study, some authors have reported a higher prevalence of MRSA in their studies like Velvizhi et al. (2011). ${ }^{19}$ Fasih et al. (2010). ${ }^{20}$ In comparison to these studies, the prevalence of MRSA in our hospital appears to be low. The prevalence of S. aureus isolates with Inducible phenotype in our study was $18.4 \%$. Similar results were obtained by Lyall et al. (2013). ${ }^{21}$ where they found $17.2 \%$ prevalence of $\mathrm{S}$. aureus isolates with Inducible phenotype. Other studies by various authors like Yilmaz et al. (2007). ${ }^{1}$ Aleksandra et al. (2014). ${ }^{22}$ Reddy et al. (2012). ${ }^{23}$ and Gadepalli et al. (2006). ${ }^{17}$ showed the prevalence of S. aureus isolates with Inducible phenotype as $19.8 \%, 16.2 \%, 20.6 \%$ and $21 \%$ respectively.

In the present study, we found that the maximum resistance in S. aureus isolates was against Penicillin 10U (92.1\%) followed by Amoxicillin-Clavulanic acid 20/10 $\mu \mathrm{g}$ (47.8\%) and Ciprofloxacin 5 $\mu \mathrm{g}$ (43.4\%).

However, all $(100 \%)$ the isolates were susceptible to Vancomycin $30 \mu \mathrm{g}$, Teicoplanin $20 \mu \mathrm{g}$ and Linezolid 30 $\mu \mathrm{g}$. The resistance pattern did not vary with different phenotypes.

But resistance to different antibiotics was significantly more amongst MRSA isolates than MSSA isolates. Similar results were found in a study done by Reddy et al. (2012). ${ }^{23}$ The prevalence of multidrug resistance amongst different phenotypes was as follows: $22.8 \%, 41.6 \%$ and $82.9 \%$ in isolates with Inducible phenotype, MS phenotype and Constitutive phenotype respectively. So the prevalence of multidrug resistance was least likely with inducible clindamycin resistance than with other phenotypes. We found $18.4 \%$ prevalence of $\mathrm{S}$. aureus isolates with inducible phenotype amongst the total S. aureus isolates obtained. Labelling all in vitro erythromycin resistant and clindamycin susceptible strains as clindamycin resistant will prevent the use of clindamycin in treating those Staphylococcal infections, which would have responded to clindamycin therapy.

On the other hand, if D-Test is not done to identify these inducible phenotype strains, we would misidentify them as clindamycin susceptible on the basis of in vitro sensitivity testing, ultimately leading to therapeutic failure. Therefore, performing D-Test on routine basis is essentially the need of the hour to differentiate between strains with Inducible phenotype and MS phenotype, so as to prevent irrational use of drugs, thereby preventing drug resistance. Hence, this simple, cheap, easy to perform in vitro D-test can be incorporated as a part of routine antibiotic susceptibility testing, so that the isolates with inducible phenotype can be identified well in time.

\section{REFERENCES}

1. Yilmaz G, Aydin K, Iskender S, et al. Detection and prevalence of inducible clindamycin resistance in staphylococci. J Med Microbiol 2007;56:342-5.

2. Fiebelkorn KR, Crawford SA, McElmeel ML, et al. Practical disc diffusion method for detection of inducible clindamycin resistance in Staphylococcus aureus and coagulase negative staphylococci. J Clin Microbiol 2003; 41:4740-4.

3. Deotale V, Mendiratta DK, Raut U, et al. Inducible clindamycin resistance in Staphylococcus aureus isolated from clinical samples. Indian J Med Microbiol 2010; 28:124-6.

4. Ajantha GS, Kulkarni RD, Shetty J, et al. Phenotypic detection of inducible clindamycin resistance among Staphylococcus aureus isolates by using the lower limit of recommended inter-disk distance. Indian J Pathol Microbiol 2008;51:376-8.

5. Weisblum B. Erythromycin resistance by ribosome modification. Antimicrob Agents Chemother 1995; 39:577-85.

6. Roberts MC, Sutcliffe J, Courvalin P, et al. Nomenclature for macrolide and macrolide-lincosamide-streptogramin B resistance determinants. Antimicrob Agents Chemother 1999;43:2823-30.

7. Brisson-Noel A, Delrieu P, Samain D, et al. Inactivation of lincosamide antibiotics in staphylococcus. Identification of lincosamide 0-nucleotidyltransferases and comparison of the corresponding resistance genes. J Biol Chem 1988;263:15880-7.

8. Adaleti R, Nakipoglu Y, Ceran N, et al. Prevalence of phenotypic resistance of Staphylococcus aureus isolates to macrolide, lincosamide, streptogramin B, ketolid and linezolid antibiotics in Turkey. Braz J Infect Dis 2010;14:11-4 
9. Leclerq R. Mechanism of resistance to macrolides and lincosamides: nature of the resistance elements and their clinical implications. Clin Infect Dis 2002;34:482-92.

10. Drinkovic D, Fuller ER, Shore KP, et al. Clindamycin treatment of Staphylococcus aureus expressing inducible resistance. J Antimicrob Chemother 2001;48:315-6.

11. Siberry GK, Tekle T, Carroll K, et al. Failure of clindamycin treatment of methicillin-resistant Staphylococcus aureus expressing inducible clindamycin resistance in vitro. Clin Infect Dis 2003;37:1257-60.

12. Watanakunakorn C. Clindamycin therapy of Staphylococcus aureus and endocarditis. Clinical relapse and development of resistance to clindamycin, lincosamide and erythromycin. Am J Med 1976;60:41925.

13. Schreckenberger PC, Ilendo E, Ristow KL. Incidence of constitutive and inducible clindamycin resistance in Staphylococcus aureus and coagulase-negative staphylococci in a community and a tertiary care hospital. J Clin Microbiol 2004;42:2777-9.

14. Staphylococcus and Micrococcus. In: Kloos WE, Banerman TL, editors. Manual of clinical microbiology, 7th ed. Washington DC: ASM Press; 1999. p. 264-82.

15. Clinical and Laboratory Standards Institute. CLSI document M100-S23. Performance standards for antimicrobial susceptibility testing; twenty-third informational supplement. Wayne, PA: Clinical and Laboratory Standards Institute; 2013.

16. Baird D. Staphylococcus: Cluster forming Gram-positive cocci. In: Collee JG, Fraser AG, Marmion BP, Simmons A, editors. Mackie \& McCartney Practical Medical Microbiology, $14^{\text {th }}$ ed. Chichester: Churchill Livingstone; 2012. p. 245-58.
17. Gadepalli R, Dhawan B, Mohanty S, et al. Inducible clindamycin resistance in clinical isolates of Staphylococcus aureus. Indian J Med Res 2006;123:5713.

18. Azap OK, Arslan H, Timurkaynak F, et al. Incidence of inducible clindamycin resistance in staphylococci: first results from Turkey. Clin Microbiol Infect 2005;11:582-4.

19. Velvizhi G, Sucilathangam G, Palaniappan N. Prevalence and phenotypic detection of erythromycin induced resistance to clindamycin in MRSA isolates. J Clin Diagn Res 2011;5:1195-8.

20. Fasih N, Irfan S, Zafar A, et al. Inducible clindamycin resistance due to expression of erm genes in Staphylococcus aureus: report from a tertiary care hospital, Karachi, Pakistan. J Pak Med Assoc 2010;60:750-3.

21. Lyall KS, Gupta V, Chinna D. Inducible clindamycin resistance among clinical isolates of Staphylococcus aureus. J Mahatma Gandhi Inst Med Sci 2013;18:112-5.

22. Aleksandra AD, Misic MS, Mira ZV, et al. Prevalence of inducible clindamycin resistance among communityassociated staphylococcal isolates in central Serbia. Indian J Med Microbiol 2014;32:49-52.

23. Reddy PS, Suresh R. Phenotypic detection of inducible clindamycin resistance among the clinical isolates of Staphylococcus aureus by using the lower limit of interdisk space. J Microbiol Biotech Res 2012;2:258-64. 